\title{
MINIMAX DESIGN OF PREFILTERS FOR MANEUVERING FLEXIBLE STRUCTURES
}

\author{
Tarunraj Singh * Yong-Lin Kuo ${ }^{\dagger}$ \\ Department of Mechanical and Aerospace Engineering \\ SUNY at Buffalo, Buffalo, New York 14260
}

\begin{abstract}
This paper focuses on the design of prefilters for maneuvering structures with the objective of desensitizing the controller to errors in the system model. Given information about the expected variation of the uncertain parameters, a minimax optimization problem is formulated to minimize the maximum value of the residual energy over the range of the uncertain parameter. The proposed technique is illustrated on a spring-mass-dashpot system with uncertainties in both the damping and stiffness constants and on a two-mass two-spring, two input system.
\end{abstract}

\section{INTRODUCTION}

Control of vibratory structures by filtering the reference input to the system has been addressed by numerous researchers [14], [7], [3], [8] etc. Singh and Singhose [2] present a tutorial related to the design of input shapers/time-delay filters and include a comprehensive list of relevant papers. Smith [14] proposed a wave cancellation technique to drive a second order system to its final position in finite time. However, this technique is sensitive to modeling errors. Singer and Seering [7] proposed a simple technique to desensitize the input shaper to modeling errors. This involved design of a sequence of impulses which forced the magnitude of the residual energy and its derivative with respect to damping or natural frequency, to zero. Singh and Vadali [8] arrived at the same results of Singer and Seering [7] by the design of a time-delay filter which cancelled the poles of the system. They also showed that by cascading the time-delay filter designed to cancel the poles of the system, the resulting filter was insensitive to errors in modeled damping and frequency. The idea of locating multiple zeros

\footnotetext{
*Associate Professor, Mechanical \& Aerospace Engineering, Member AIAA

${ }^{\dagger}$ Graduate Student, Mechanical \& Aerospace Engineering
}

of a time-delay filter at the estimated location of the poles of the system has been exploited to design robust time-optimal control [9], [5], robust fuel-time optimal control [11], fuel constrainted time-optimal control [12] etc. Liu and Singh [4] extended this idea to nonlinear systems undergoing rest-to-rest maneuvers, by requiring the sensitivity of the system states with respect to uncertain parameters be zero at the final time.

Techniques to increase the range of uncertain parameters where the residual vibration is below a prespecified amount has been addressed by Singhose et al. [13]. This was referred to as the extra insensitive input shaper. Pao et al. [6] included the probability distribution of the uncertain parameters into the design process to arrive at input shapers which weighted the nominal value of the uncertain parameter the most.

This paper proposes a technique to design timedelay filters which minimize the maximum magnitude of the residual vibration over the range of the uncertain parameter. Closed form expressions for the analytical gradients of the cost function and constraints have been derived by Singh [10], which can be used to expedite the convergence of optimization algorithms for the minimax time-delay filters, studied in this paper. The resulting controller will be referred to as the minimax time-delay controller. The first section will review the development of the time-delay control. This will be followed by the development of the minimax timedelay controller which is illustrated on single input numerical example. The penultimate section presents a multi-input example and final section summarizes results generated in this paper.

\section{TIME-DELAY CONTROL}

This section reviews the time-delay control technique. Figure 1 represents the time-delay control of a second order underdamped system. The parameters $A_{0}$ and $T$ need to be determined so that the poles of the system are cancelled by a pair of zeros of the timedelay filter. The location of the zeros of the time-delay 


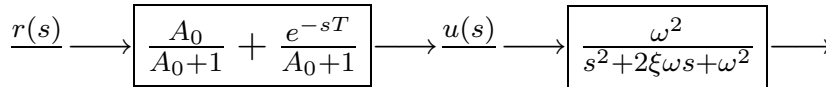

Fig. 1 Single Time-Delay Controlled System

filter are given by the solution of the equation

$$
A_{0}+e^{-s T}=0
$$

where $s$ is the Laplace variable. To locate a pair of zeros of the time-delay filter at

$$
s=-\zeta \omega \pm j \omega \sqrt{1-\zeta^{2}},
$$

we can show that

$$
A_{0}=\exp \left(\frac{\zeta \pi}{\sqrt{1-\zeta^{2}}}\right)
$$

and

$$
T=\frac{\pi}{\omega_{n} \sqrt{1-\zeta^{2}}}
$$

where $\zeta$ is the damping ratio and $\omega$ is the natural frequency of the system.

The single time-delay control, by cancelling the poles corresponding to the oscillatory behavior of the system, provides us with a technique to produce nonoscillatory response. The cancellation of the poles of the system is contingent on the availability of accurate estimates of the pole locations. To improve the robustness of the time-delay control to errors in estimated location of the poles, the two time-delay controller is proposed whose transfer function is

$$
A_{0}+A_{1} e^{-s T}+e^{-2 s T} .
$$

The requirement of cancelling the poles of the system results in two constraints. The third constraint is derived by requiring the derivative of Equation 5 with respect to $\sigma$ or $\omega$ be forced to zero. Solving the three equations results in

$$
\begin{aligned}
& A_{0}=\exp \left(\frac{2 \zeta \pi}{\sqrt{1-\zeta^{2}}}\right) \\
& A_{1}=\exp \left(\frac{\zeta \pi}{\sqrt{1-\zeta^{2}}}\right)
\end{aligned}
$$

and

$$
T=\frac{\pi}{\omega \sqrt{1-\zeta^{2}}}
$$

It can be seen from the parameters of the two timedelay controller that it can be derived by cascading $y$ (stwo, single time-delay controllers. This now provide the designer with a simple technique to desensitize the time-delay controller to modeling errors.

\section{MINIMAX TIME-DELAY CONTROL}

With the knowledge that the uncertain parameters lie within a specified range, it is desirable to design a controller with the worst model in mind. This can be achieved by considering the performance of the controller over the range of the uncertain parameter. In this paper, a design technique is proposed, which minimizes the worst performance of the system. The metric used to gauge the performance of the system corresponds to the residual energy of the system at the end of the maneuver. The goal of the optimization problem is to minimize the maximum magnitude of the residual energy over the entire range of the uncertain parameters.

For an asymptotically stable mechanical system undergoing rest-to-rest maneuvers, the model can be represented as

$$
M \ddot{y}+C(p) \dot{y}+K(p) y=D r
$$

where $M$, and $K$ are positive definite matrices and $C$ is positive semi-definite. $p$ is a vector of uncertain parameters and is bounded by the lower $p_{i}^{l b}$ and upper $p_{i}^{u b}$ bounds:

$$
p_{i}^{l b} \leq p_{i} \leq p_{i}^{u b}
$$

The objective is to design a time-delay filter which pre-filters the reference input $r$ to the system with the objective of

$$
\min _{T_{i}, A_{i}} \max _{p} \sqrt{\frac{1}{2} \dot{y}^{T} M \dot{y}+\frac{1}{2}\left(y-y_{f}\right)^{T} K\left(y-y_{f}\right)}
$$

where $T_{i}$ and $A_{i}$ are parameters which define the robust time-delay filter and $y_{f}$ corresponds to the final displacement states of the system. The above equation will be referred to as the pseudo-energy function since it is associated with a hypothetical spring whose potential energy is zero when $y=y_{f}$. Without loss of generality, we can assume that the initial displacement states are zero.

\section{NUMERICAL EXAMPLES}

The proposed technique will be illustrated on a rest-to-rest maneuver of a single mode system whose dynamics are defined by the equation 


$$
m \ddot{y}+c \dot{y}+k y=k r
$$

with the boundary conditions

$$
y(0)=\dot{y}(0)=0, y\left(t_{f}\right)=1, \dot{y}\left(t_{f}\right)=0
$$

where $t_{f}$ is the maneuver time.

\section{ONE UNCERTAIN PARAMETER}

First, a minimax time-delay controller will be designed assuming that only $k$ is uncertain and satisfies the constraint

$$
0.7 \leq k \leq 1.3
$$

where the nominal value of $k=1$ and $m=1, c=$ 0.2. The form of the transfer function for the minimax time-delay controller is chosen to be

$$
A_{0}+A_{1} e^{-s T}+A_{2} e^{-2 s T}
$$

which is identical to the robust time-delay controller (Equation 5). The optimization problem can be stated as the determination of $A_{0}, A_{1}, A_{2}$ and $T$ of the timedelay filter so as to

$$
\min _{A_{i}, T} \max _{k}\left(\frac{1}{2} m \dot{y}^{2}+\frac{1}{2} k(y-1)^{2}\right)
$$

The transfer function of the non-robust time-delay filter for the nominal system is

$$
0.5783+0.4217 e^{-3.1574 s} .
$$

With the knowledge that two non-robust filters in cascade will force the derivative of the square root of the pseudo-energy to be zero at the nominal value of the system parameters resulting in smaller magnitude of residual vibration in the vicinity of the nominal parameters as illustrated in Figure 2 (dashed line), the transfer function of the robust time-delay controller can be shown to be

$$
0.3344+0.4877 e^{-3.1574 s}+0.1788 e^{-6.3148 s} .
$$

The parameters of the time-delay filter (Equation 18), will be used as initial guesses for the minimax algorithm. The optimization toolbox of MATLAB ${ }^{\circledR}$ is used to solve the minimax optimization algorithm. An iterative approach proposed by Mills and Bryson [1] is used to solve for the minimax solution. This technique

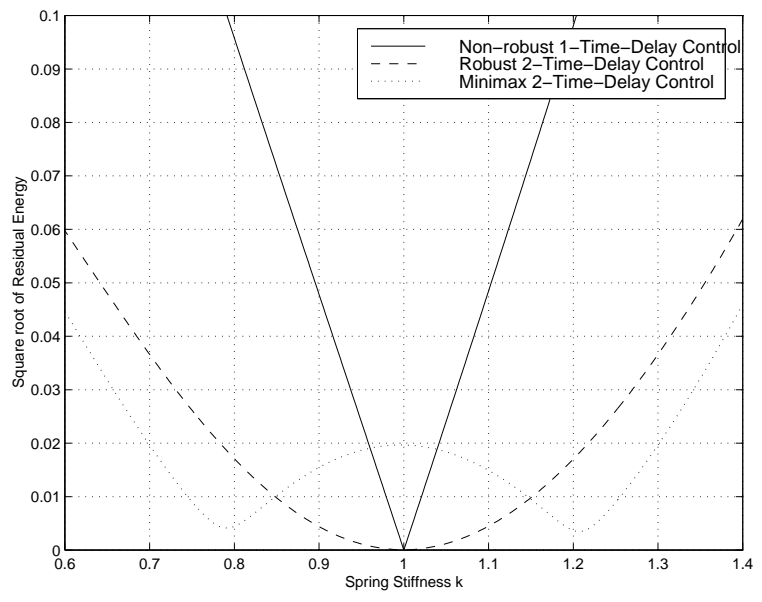

Fig. 2 Residual Vibration Distribution

entails initiating the optimization algorithm with tight bounds on the parameters to be optimized for and gradually relaxing the bounds to their problem defined bounds, iteratively. The solution of every iteration is used as the initial condition for the next. The optimal minimax time-delay filter is given by the transfer function

$$
0.3452+0.4730 e^{-3.1703 s}+0.1818 e^{-6.2060 s} .
$$

Figure 2 (dotted line) illustrate the variation of the residual energy of the system as a function of the uncertain parameter $k$. It can be seen that the maximum magnitude of the residual energy in the range of the uncertain parameters occurs at the bounding limits $(k$ $=0.7, k=1.3$ ) and near the nominal value of $k$. It is also clear that the maximum magnitude of the residual energy is significantly smaller than that resulting from the robust time-delay filter defined by equation 18 over the entire range of $k$. However, near the nominal value of $k$, the minimax solution has the largest magnitude of residual vibration. The minimax solution can also be shown to be similar to the extra-insensitive input shaper proposed by Singhose et al. [13] where an optimization problem is formulated by defining the magnitude of residual vibration permitted at the nominal value of the uncertain parameter and solving for the magnitudes of a sequence of impulses. The impulse sequence is required to satisfy the constraints that the magnitude of the residual vibration is zero at two frequencies which flank the nominal value and the slope of the residual energy distribution curve is zero at the nominal value of the uncertain parameter.

Notwithstanding that the maximum magnitude of the residual vibration over the range of possible value 
of $k$ has been minimized, the fact that the residual vibration near the nominal value of $k$ is the maximum is a drawback of this controller. To address the aforementioned disadvantage, an additional constraint is included into the minimax optimization problem which requires the magnitude of the residual vibration to be zero at the nominal value of the uncertain parameter. The added constraint necessitates addition of a timedelay to the time-delay filter defined by Equation 19, resulting in the transfer function

$$
A_{0}+A_{1} e^{-s T}+A_{2} e^{-2 s T}+A_{3} e^{-3 s T} \text {. }
$$

The unknown parameters of Equation 20 are solved for using the solution of the parameters of three nonrobust time-delay filters in cascade as initial guesses. The transfer function of the minimax time-delay controller with the constraint to force the residual vibration to be zero at the nominal value of $k$ can be shown to be

$$
\begin{gathered}
0.2052+0.4141 e^{-3.1652 s} \\
+0.3015 e^{-6.3304 s}+0.07924 e^{-9.4956 s} .
\end{gathered}
$$

Figure 3 illustrates the distribution of the residual energy of the time-delay filter designed by cascading three non-robust time-delay filters (solid line) and the minimax time-delay filter (dashed line).

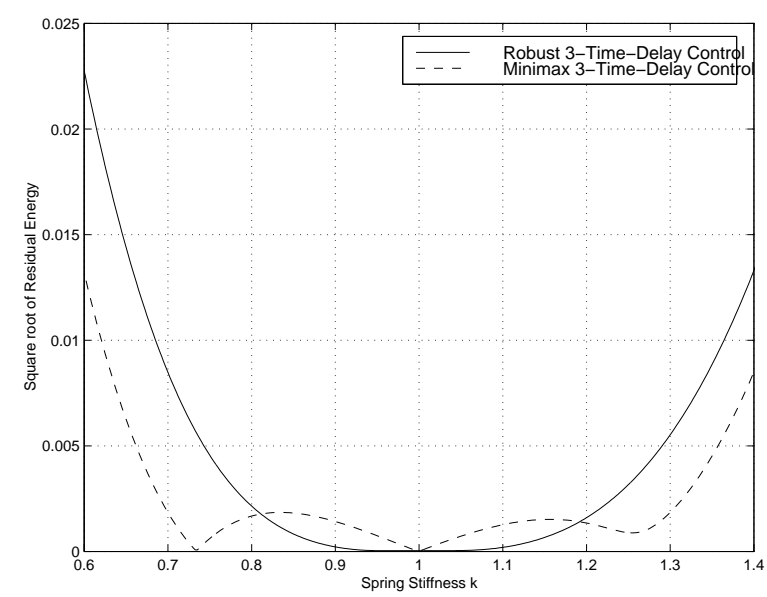

Fig. 3 Residual Vibration Distribution

\section{TWO UNCERTAIN PARAMETERS}

Equation 12 is considered again and a minimax time-delay controller is designed assuming that the two uncertain parameters satisfy the constraints

$$
0.7 \leq k \leq 1.3,0.1 \leq c \leq 0.3
$$

where the nominal values of $k$ and $c$ are 1 and 0.2 respectively. A time-delay filter of the form

$$
A_{0}+A_{1} e^{-s T_{1}}+A_{2} e^{-2 s T}
$$

is selected which is identical to Equation 15. An optimization problem is solved which minimizes the maximum value of the residual energy at time $2 T$ of a set of $(k, c)$ parameters over the uncertain region given by Equation 22. The optimal time-delay filter transfer function is

$$
0.3380+0.4732 e^{-3.1541 s}+0.1888 e^{-6.3082 s} .
$$

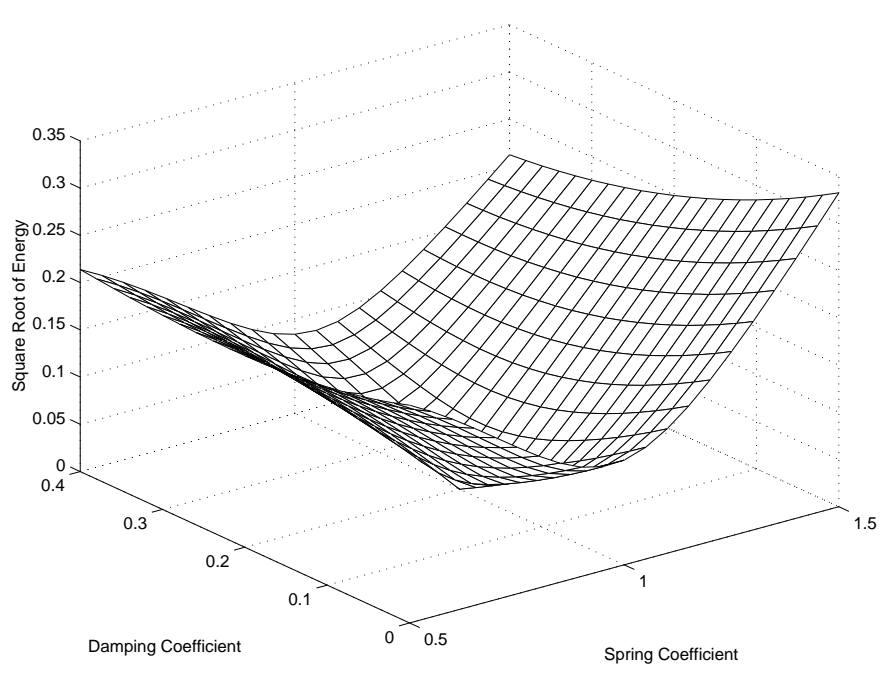

Fig. 4 Residual Energy Distribution (NonRobust Control)

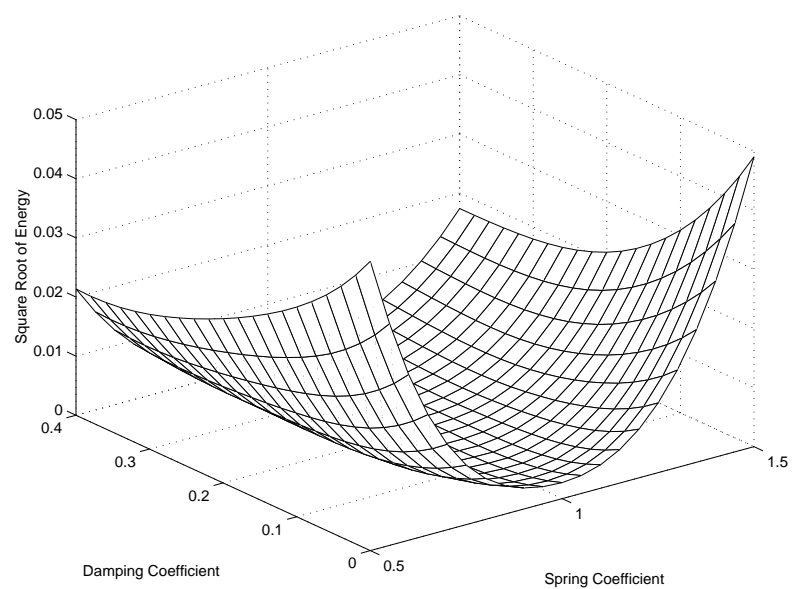

Fig. 5 Residual Energy Distribution (Robust Control)

Figure 4 illustrates the variation of the residual energy as a function of damping and stiffness constants 
for the time-delay filter given by Equation 17 which was designed to cancel the poles of the system based on the nominal values of the uncertain parameters. It is clear that the increase in the residual vibration is significant as the modeling error increases. Figure 5 exemplifies the variation of the residual energy when the robust time-delay filter (Equation 18) is used to prefilter the step input to the system. The minimax time-delay filter given by Equation 24 illustrates that the residual energy over the uncertain region is greatly reduced. However, this is at the cost of increasing the residual vibration at the nominal value of the uncertain parameters as shown in Figure 6. This can be remedied by adding a time-delay to the above filter and including a constraint into the optimization problem, which forces the residual energy to be zero at the nominal value of the model parameters. The resulting filter transfer function is

$$
\begin{gathered}
0.1963+0.4122 e^{-3.1664 s} \\
+0.3091 e^{-6.3328 s}+0.08243 e^{-9.4992 s} .
\end{gathered}
$$

Figure 7 illustrates that the residual vibration over the uncertain region has been further reduced and is zero at the nominal value of the model parameters.

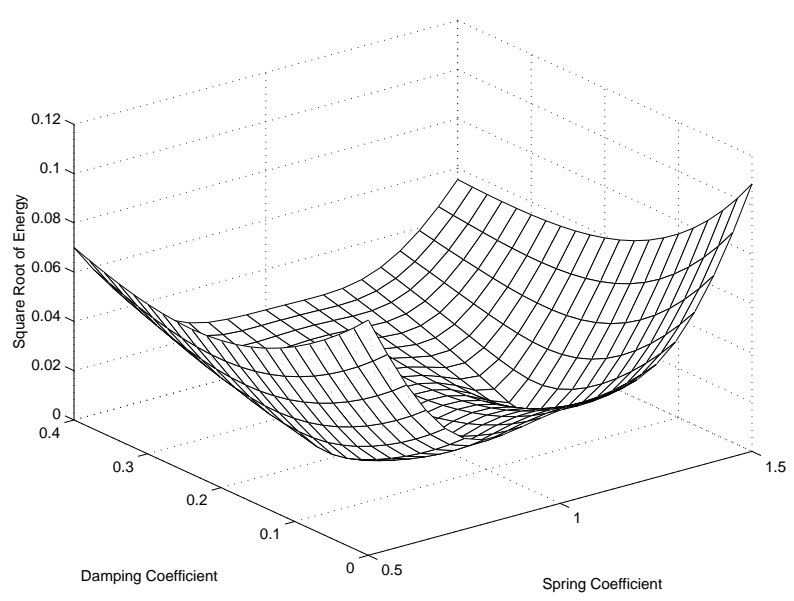

Fig. 6 Residual Energy Distribution (Minimax Control)

\section{MULTI INPUT SYSTEMS}

The technique for the design of minimax prefilters can be extended to multi-input systems. Figure 8 illustrates a two mass/spring system with two inputs. The equations of motion of the system are

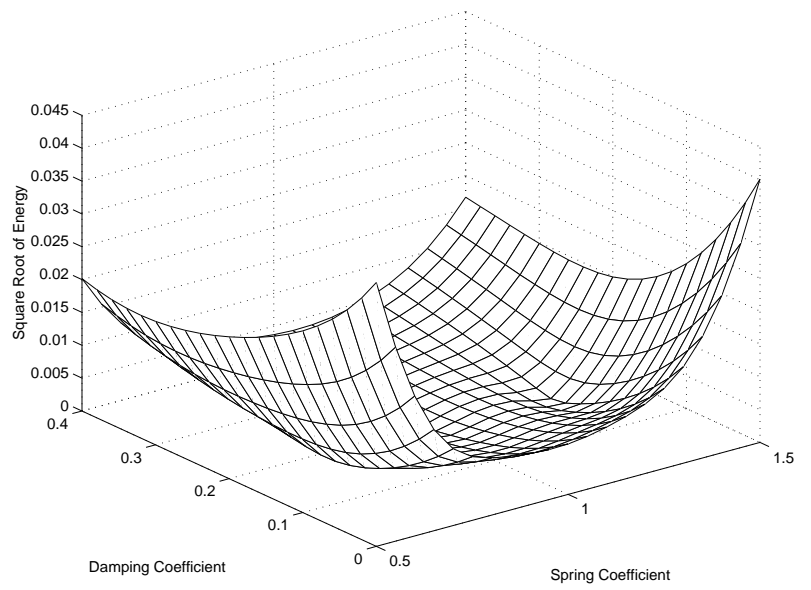

Fig. 7 Residual Energy Distribution (Minimax Control with Zero Vibration Constraint)

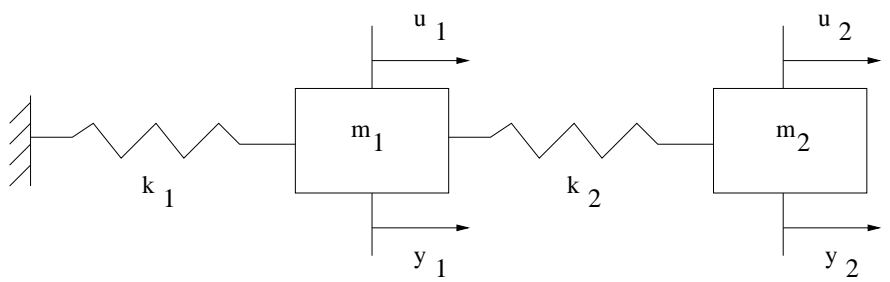

Fig. 8 Two Input Oscillator

$$
\begin{array}{r}
{\left[\begin{array}{cc}
m_{1} & 0 \\
0 & m_{2}
\end{array}\right]\left\{\begin{array}{l}
\ddot{y}_{1} \\
\ddot{y}_{2}
\end{array}\right\}+\left[\begin{array}{cc}
k_{1}+k_{2} & -k_{2} \\
-k_{2} & k_{2}
\end{array}\right]\left\{\begin{array}{l}
y_{1} \\
y_{2}
\end{array}\right\}=} \\
{\left[\begin{array}{ll}
1 & 0 \\
0 & 1
\end{array}\right]\left\{\begin{array}{l}
u_{1} \\
u_{2}
\end{array}\right\} .}
\end{array}
$$

The objective is to design a time-delay filter with the objective of completing a rest-to-rest maneuver with the boundary conditions:

$$
\begin{array}{r}
y_{1}(0)=y_{2}(0)=\dot{y}_{1}(0)=\dot{y}_{2}(0)=0 \\
y_{1}\left(t_{f}\right)=2, y_{2}\left(t_{f}\right)=1, \dot{y}_{1}\left(t_{f}\right)=\dot{y}_{2}\left(t_{f}\right)=0,
\end{array}
$$

and which it is robust to uncertainties in the spring stiffness $k_{1}$ and $k_{2}$. It is assumed that the uncertain parameters lie in the range:

$$
0.7 \leq k_{1} \leq 1.3 \text { and } 0.7 \leq k_{2} \leq 1.3 .
$$

The control input is rewritten as

$$
\left\{\begin{array}{l}
u_{1} \\
u_{2}
\end{array}\right\}=\left.\left[\begin{array}{cc}
k_{1}+k_{2} & -k_{2} \\
-k_{2} & k_{2}
\end{array}\right]\right|_{\text {nominal }}\left\{\begin{array}{l}
v_{1} \\
v_{2}
\end{array}\right\}
$$


The final values of the new control inputs to satisfy the boundary conditions are given as

$$
\left\{\begin{array}{l}
v_{1} \\
v_{2}
\end{array}\right\}=\left\{\begin{array}{l}
2 \\
1
\end{array}\right\} \text {. }
$$

Assuming the nominal values of the spring stiffness are $k_{1}=1, k_{2}=1$, the final values of the control inputs are $u_{1}=3, u_{2}=-1$. A minimax problem is formulated to minimize the maximum magnitude of the residual energy of the system over the domain of uncertainty defined by Equation 29. The transfer function of the time-delay filters are parameterized as

$$
\begin{aligned}
G_{1}(s) & =\sum_{i=0}^{N} A_{1 i} \exp \left(-s T_{1 i}\right) \text { where } T_{10}=0 \\
G_{2}(s) & =\sum_{i=0}^{N} A_{2 i} \exp \left(-s T_{2 i}\right) \text { where } T_{20}=0 .
\end{aligned}
$$

The minimax optimization problem is used to solve for $A_{1 i}, T_{1 i}, A_{2 i}$, and $T_{2 i}$. The following constraints are imposed on the optimization problem. To ensure that the systems states are quiesent at the final time, and since the system modes cannot be decoupled, we require

$$
T_{1 N}=T_{2 N}
$$

Further, we require

$$
\left\{\begin{array}{l}
\sum_{i=0}^{N} A_{1 i} \\
\sum_{i=0}^{N} A_{2 i}
\end{array}\right\}=\left\{\begin{array}{l}
y_{1}\left(t_{f}\right) \\
y_{2}\left(t_{f}\right)
\end{array}\right\}
$$

where $t_{f}$ is the final maneuver time.

To compare the performance of the minimax timedelay filter to conventional filters, two classes of filters are considered. The first is designed to cancel the undamped poles of the system which is given by the time-delay filters

$$
\begin{aligned}
G_{1}(s)=2\left(\frac{1}{2}+\frac{1}{2} \exp \left(-s \frac{\pi}{\omega_{1}}\right)\right) \\
\left(\frac{1}{2}+\frac{1}{2} \exp \left(-s \frac{\pi}{\omega_{2}}\right)\right) \\
G_{2}(s)=1\left(\frac{1}{2}+\frac{1}{2} \exp \left(-s \frac{\pi}{\omega_{1}}\right)\right) \\
\left(\frac{1}{2}+\frac{1}{2} \exp \left(-s \frac{\pi}{\omega_{2}}\right)\right)
\end{aligned}
$$

where $\omega_{1}$ and $\omega_{2}$ are the nominal frequencies of the controlled system. Next, a minimax problem is solved for the same number of delays as in Equations 36 and 37. The optimal parameters of the minimax time-delay filter are given in Table 1.

\begin{tabular}{|c|c|c|c|c|}
\hline \hline$A_{1 i}$ & 0.5200 & 0.5954 & 0.5344 & 0.3503 \\
\hline$T_{1 i}$ & 0.0 & 3.3688 & 6.3719 & 12.1535 \\
\hline$A_{2 i}$ & 0.3630 & -0.5139 & 0.7001 & 0.4508 \\
\hline$T_{2 i}$ & 0 & 1.6853 & 6.8284 & 12.1535 \\
\hline
\end{tabular}

Table 1 Minimax Time-Delay Filter Parameters

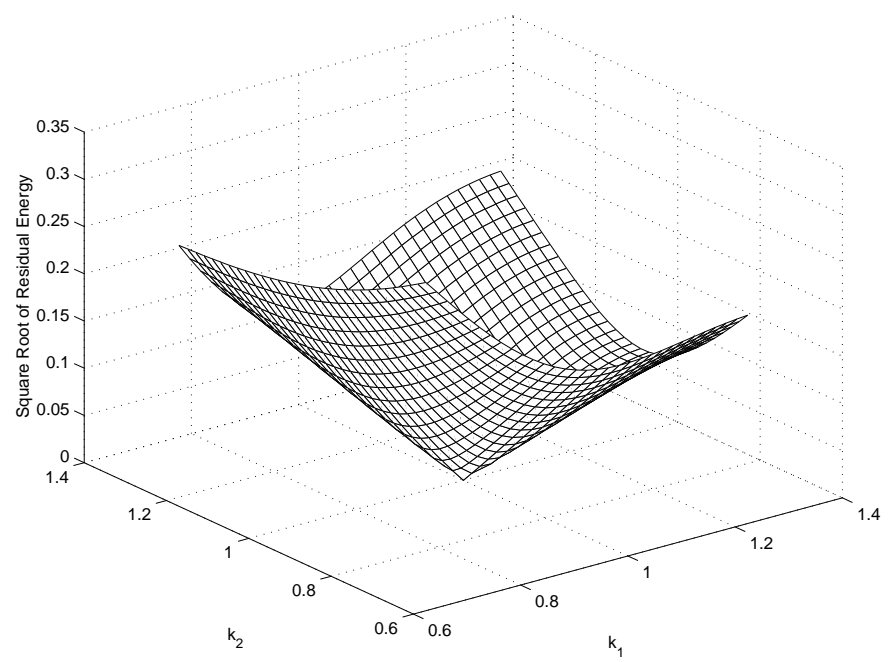

Fig. 9 Residual Vibration Distribution (Nonrobust Filter)

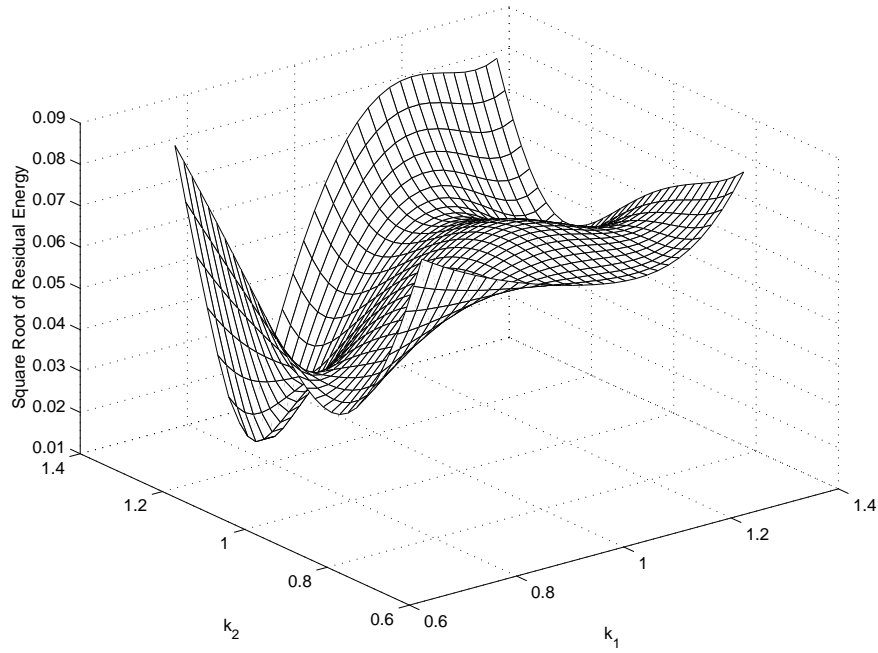

Fig. 10 Residual Vibration Distribution (Minimax Filter)

Figure 9 and 10 illustrate the variation of the residual energy of the nonrobust and the corresponding minimax prefilters. It is clear that for the nominal model, the non-robust filter results in zero residual energy. However, over the entire uncertain region, the minimax filter outperforms the nonrobust filter. 
The second is the robust time-delay filter designed by locating multiple zeros of the time-delay filter at the estimated location of the poles of the system, which is given by the transfer functions:

$$
\begin{aligned}
G_{1}(s)=2\left(\frac{1}{4}+\right. & \left.\frac{1}{2} \exp \left(-s \frac{\pi}{\omega_{1}}\right)+\frac{1}{4} \exp \left(-2 s \frac{\pi}{\omega_{1}}\right)\right) \\
& \left(\frac{1}{4}+\frac{1}{2} \exp \left(-s \frac{\pi}{\omega_{2}}\right)+\frac{1}{4} \exp \left(-2 s \frac{\pi}{\omega_{2}}\right)\right) \\
G_{2}(s)=1( & \left(\frac{1}{4}+\frac{1}{2} \exp \left(-s \frac{\pi}{\omega_{1}}\right)+\frac{1}{4} \exp \left(-2 s \frac{\pi}{\omega_{1}}\right)\right) \\
& \left(\frac{1}{4}+\frac{1}{2} \exp \left(-s \frac{\pi}{\omega_{2}}\right)+\frac{1}{4} \exp \left(-2 s \frac{\pi}{\omega_{2}}\right)\right)
\end{aligned}
$$

Next, a minimax time-delay filter is designed to minimize the maximum magnitude of the residual energy of the uncertain domain and the optimal parameters of the time-delay filter are given in Table 2 .

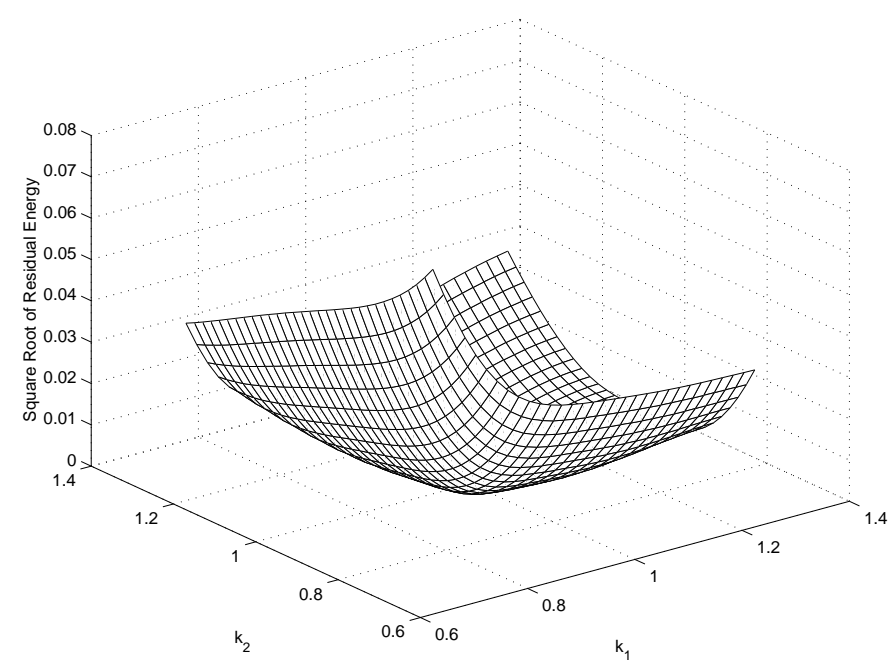

Fig. 11 Residual Vibration Distribution (Robust Control)

From Figures 11 and 12, it can be seen that the maximum magnitude of the square root of the residual energy over the uncertain region reduces from 0.0762 to 0.0116 a $85 \%$ reduction.

\section{CONCLUSIONS}

This paper proposes a simple technique to design time-delay filters for reducing the maximum magnitude of residual vibration over a range of parameter

\begin{tabular}{|c|c|c|c|c|c|c|c|c|c|}
\hline \hline$A_{1 i}$ & 0.1014 & 0.1568 & 0.3376 & 0.2147 & 0.2609 & 0.2843 & 0.3379 & 0.2127 & 0.0938 \\
\hline$T_{1 i}$ & 0 & 2.2897 & 4.7851 & 7.0169 & 8.0249 & 10.2515 & 12.2812 & 14.8886 & 18.6908 \\
\hline$A_{2 i}$ & 0.1003 & -0.0867 & 0.1551 & 0.0678 & 0.1571 & -0.1312 & 0.3658 & 0.2104 & 0.1613 \\
\hline$T_{2 i}$ & 0 & 5.0907 & 5.0907 & 6.6232 & 8.2987 & 11.6788 & 11.8754 & 15.1427 & 18.6908 \\
\hline
\end{tabular}

Table 2 Minimax Time-Delay Filter Parameters

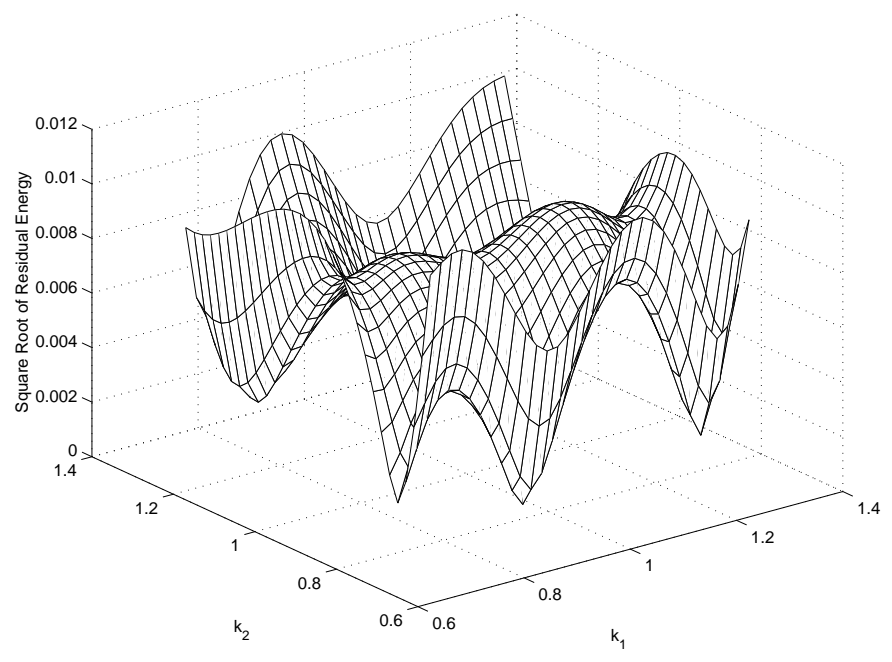

Fig. 12 Residual Vibration Distribution (Minimax Control)

uncertainty. A minimax optimization problem is formulated which requires only one equations which is used both as the cost function and when evaluated at the nominal value of the model parameters, as the constraint. The minimax filter is designed for a springmass-dashpot with uncertainties in the spring stiffness and damping factor of the systems. This is followed by a two-mass two-spring, two-input system, with uncertainties in the stiffness of both the springs. 


\section{REFERENCES}

${ }^{1}$ Bryson, A. E., and Mills, R. A., "Linear-QuadraticGaussian Controllers with Specified Parameter Robustness", AIAA Journal of Guidance, Control and Dynamics , Vol. 21, No. 1, 1998, pp 11-18.

${ }^{2}$ Singh, T., and Singhose, W., E., 'Tutorial on Input Shaping/Time Delay Control of Maneuvering Flexible Structures"", Proceedings of the American Control Conference, Anchorage, Al, May 8-10, 2002.

${ }^{3}$ Junkins J. L., Rahman, Z., Bang, H., "Near-Minimum Time Maneuvers of Flexible Vehicles: A Liapunov Control Law Design Method", Mechanics and Control of Large Flexible Structures, AIAA Publication, Washington, DC, 1990.

${ }^{4}$ Liu, S-W., and Singh, T., "Robust Time-Optimal Control of Nonlinear Structures with Parameter Uncertainties", ASME Journal of Dynamic Systems, Measurement and Control, Vol. 119, No. 4, 1997, pp 743-748.

${ }^{5}$ Liu, Q. and Wie, B., "Robust Time-Optimal Control of Uncertain Flexible Spacecraft", AIAA Journal of Guidance, Control and Dynamics, Vol. 15, No. 3, 1992, pp 597-604.

${ }^{6}$ Pao, L. Y., Chang, T. N., and Hou, E., "Input Shaper Design for Minimizing the Expected Level of Residual Vibration in Flexible Structures", Proceedings of the American Control Conference, Albuquerque, NM, 1997.

${ }^{7}$ Singer, N. C, and Seering, W. P., "Preshaping Command Inputs to Reduce System Vibrations", ASME Journal of Dynamic Systems, Measurement and Control, vol. 115, 1990, pp 76-82.

${ }^{8}$ Singh, T. and Vadali, S. R., "Robust Time-Delay Control", ASME Journal of Dynamic Systems, Measurement and Control, Vol. 115, No 2(A), 1993, pp 303-306.

${ }^{9}$ Singh, T. and Vadali, S. R., "Robust Time-Optimal Control: Frequency Domain Approach", AIAA Journal of Guidance, Control and Dynamics, Vol. 17, No. 2, 1994, pp 346-353.

${ }^{10}$ Singh, T., "Minimax Design of Robust Controllers for Flexible Systems", To appear in the AIAA Journal of Guidance, Control and Dynamics.

${ }^{11}$ Singh, T., "Fuel/Time Optimal Control of the Benchmark Problem", AIAA Journal of Guidance, Control and Dynamics, Vol. 18, No. 6, 1995, pp 1225-1231.

${ }^{12}$ Singhose, W., Singh, T. and Seering, W. P., "On-Off Control of Flexible Spacecraft with Specified Fuel Usage", ASME Journal of Dynamic Systems, Measurement and Control, Vol. 121, No. 2, 1999, pp 206-121.

${ }^{13}$ Singhose, W., E., Porter, L., J., and Singer, N. P., "Vibration Reduction Using Multi-Hump Extra-Insensitive Input Shapers", Proceedings of the American Control Conference, 1995.

${ }^{14}$ Smith, O. J. M., "Posicast Control of Damped Oscillatory Systems", Proceedings of the IRE, September, 1957, pp 1249-1255. 